\title{
Ferromagnetic van der Waals crystal $\mathrm{VI}_{3}$
}

\author{
Shangjie Tian, ${ }^{1, \dagger}$ Jian-Feng Zhang, ${ }^{1, \dagger}$ Chenghe Li, ${ }^{1}$ Tianping Ying, ${ }^{2}$ \\ Shiyan $\mathrm{Li}^{2,3}$ Xiao Zhang, ${ }^{4, *}$ Kai Liu, ${ }^{1, *}$ and Hechang Lei ${ }^{1, *}$ \\ ${ }^{1}$ Department of Physics and Beijing Key Laboratory of Opto-electronic Functional Materials \& Micro-nano Devices, \\ Renmin University of China, Beijing 100872, China \\ 2 State Key Laboratory of Surface Physics, Department of Physics, \\ and Laboratory of Advanced Materials, Fudan University, Shanghai 200438, China \\ 3 Collaborative Innovation Center of Advanced Microstructures, Nanjing 210093, China \\ ${ }^{4}$ State Key Laboratory of Information Photonics and Optical Communications \& School of Science, \\ Beijing University of Posts and Telecommunications, Beijing 100876, China
}

(Dated: December 18, 2018)

\begin{abstract}
We report structural, physical properties and electronic structure of van der Waals (vdW) crystal $\mathrm{VI}_{3}$. Detailed analysis reveals that $\mathrm{VI}_{3}$ exhibits a structural transition from monoclinic $C 2 / \mathrm{m}$ to rhombohedral $R \overline{3}$ at $T_{s} \sim 79 \mathrm{~K}$, similar to $\mathrm{CrX}_{3}(\mathrm{X}=\mathrm{Cl}, \mathrm{Br}, \mathrm{I})$. Below $T_{s}$, a long-range ferromagnetic $(\mathrm{FM})$ transition emerges at $T_{c} \sim 50 \mathrm{~K}$. The local moment of $\mathrm{V}$ in $\mathrm{VI}_{3}$ is close to the high-spin state $\mathrm{V}^{3+}$ ion $(S=1)$. Theoretical calculation suggests that $\mathrm{VI}_{3}$ may be a Mott insulator with the band gap of about $0.84 \mathrm{eV}$. In addition, $\mathrm{VI}_{3}$ has a relative small interlayer binding energy and can be exfoliated easily down to few layers experimentally. Therefore, $\mathrm{VI}_{3}$ is a candidate of two-dimensional FM semiconductor. It also provides a novel platform to explore 2D magnetism and vdW heterostructures in $S=1$ system.
\end{abstract}

\section{INTRODUCTION}

Two-dimensional (2D) materials have induced great interest since the discovery of graphene $\underline{1}$ They exhibit various exotic physical properties, such as enhanced directgap semiconductor in monolayer $\mathrm{MoS}_{2} \stackrel{2}{=}$ Ising superconductivity in ion-gated $\mathrm{MoS}_{2}$ and atomic layer $\mathrm{NbSe}_{2}, \frac{3,4}{,}$ robust in-plane ferroelectricity in atomic-thick SnTe, and so on. In theory, the long-range 2D magnetic order is strongly suppressed above zero temperature for isotropic Heisenberg model because the fluctuation is so strong that will destroy the spontaneous symmetry breaking (Merin-Wagner theorem) $\underline{\underline{6}}$ But when magnetic anisotropy is included, the $2 \mathrm{D}$ ferromagnetism can be stabilized and it has been realized in monolayer $\mathrm{Cr}_{2} \mathrm{Ge}_{2} \mathrm{Te}_{6}$ and $\mathrm{CrI}_{3}$ just in recent $\underline{7,8}$ These $2 \mathrm{D}$ ferromagnets provide unique opportunities for understanding, exploring and utilizing novel low-dimensional magnetism. Because of its thickness of one or few unit cells, it may be able to control the 2D magnetic properties by applying electric field, light or strain. Such magneto-electrical, magneto-optical, or spin-lattice coupling effects are usually difficult to realize in bulk crystals. For instance, recent studies show that the electrostatic doping can control the magnetism in atomically-thin $\mathrm{CrI}_{3}$ and $\mathrm{Fe}_{3-x} \mathrm{GeTe}_{2}$ flakes, leads to the dramatic enhancement of $T_{c}$ from about $100 \mathrm{~K}$ to room temperature for the latter $\stackrel{9,10}{ }$ Moreover, the weak van der Waals (vdW) interlayer coupling allows to design heterostructures between these 2D magnets and other vdW materials without considering lattice mismatch. For example, in the vdW heterostructures formed by an ultrathin $\mathrm{CrI}_{3}$ and a monolayer $\mathrm{WSe}_{2}$, the $\mathrm{WSe}_{2}$ photoluminescence intensity strongly depends on the relative alignment between photoexcited spins in $\mathrm{WSe}_{2}$ and the $\mathrm{CrI}_{3}$ magnetization. 11

Besides $\mathrm{Cr}_{2} \mathrm{Ge}_{2} \mathrm{Te}_{6}$ and $\mathrm{CrI}_{3}, \mathrm{CrSiTe}_{3}$ and $\mathrm{CrX}_{3}(\mathrm{X}=$
$\mathrm{Cl}$ and $\mathrm{Br}$ ) vdW crystals are also easy to be exfoliated and predicted to be a 2D ferromagnetic (FM) semiconductors for monolayers. ${ }^{12}$-14 Until now, however, such magnetic vdW materials are still scarce and they are mainly chromium compounds with high spin $\mathrm{Cr}^{3+}(S=3 / 2)$. In recent, the $2 \mathrm{D}$ ferromagnetism has been predicted in $\mathrm{VX}_{3}(\mathrm{X}=\mathrm{Cl}$ and $\mathrm{I})$ with $S=1.15$ It is found that $\mathrm{VX}_{3}$ is cleavable and the calculated Curie temperature $T_{c}$ is 80 and $98 \mathrm{~K}$ for $\mathrm{VCl}_{3}$ and $\mathrm{VI}_{3}$ monolayers, respectively, higher than those in $\mathrm{CrX}_{3}$.

In this work, we grow $\mathrm{VI}_{3}$ single crystals successfully and carry out a comprehensive study on their structure, physical properties and electronic structure. $\mathrm{VI}_{3}$ exhibits a structural transition from monoclinic to rhombohedral at $T_{s} \sim 79 \mathrm{~K}$ and then a long-range $\mathrm{FM}$ ordering appears at $T_{c} \sim 50 \mathrm{~K}$. Moreover, $\mathrm{VI}_{3}$ should be a Mott insulator and can be exfoliated easily to few layers.

\section{METHODS}

Single Crystal Growth. Single crystals of $\mathrm{VI}_{3}$ were grown by chemical vapor transport method. Vanadium powder $(99.99 \%)$ and iodine flake $(99.999 \%)$ in 1 : 3 molar ratio were put into a silica tube with the length of $200 \mathrm{~mm}$ and the inner diameter of $14 \mathrm{~mm}$. The tube was evacuated down to $10^{-2} \mathrm{~Pa}$ and sealed under vacuum. The tubes were placed in two-zone horizontal tube furnace and the source and growth zones were raised to 923 $\mathrm{K}$ and $823 \mathrm{~K}$ for 3 days, and then held there for 7 days. The shiny black plate-like crystals with lateral dimensions up to several millimeters can be obtained. Because the $\mathrm{VI}_{3}$ is hygroscopic, the samples were stored in glovebox in order to avoid degradation of crystals.

X-ray Crystallography. Single crystal X-ray diffraction (XRD) patterns at $40 \mathrm{~K}, 60 \mathrm{~K}$ and $100 \mathrm{~K}$ were col- 
lected using a Bruker D8 VENTURE PHOTO II diffractometer with multilayer mirror monochromatized Mo K $\alpha$ $(\lambda=0.71073 \AA)$ radiation. Unit cell refinement and data merging were done with the SAINT program, and an absorption correction was applied using Multi-Scans. At $T=100 \mathrm{~K}$, an inspection of the systematic absences immediately showed the structure to be $C$ centered. In XPrep, the suggested possible space group is $C 2, C 2 / \mathrm{m}$ and $\mathrm{Cm}$ with the value of combined figure of merit (CFOM) 11.86, 2.01 and 14.71, respectively. The mean value of $\left|E^{2}-1\right|$ (1.067) is more close to the value for a centrosymmetric space group (0.968) than that for a noncentrosymmetric one (0.736), suggesting the centrosymmetric structure of $\mathrm{VI}_{3}$. Finally the space group $C 2 / \mathrm{m}$ with the smallest CFOM value is selected. When $T=40$ $\mathrm{K}$, an inspection of the systematic absences immediately showed the structure to be $R$ lattice. The possible space group is $R \overline{3}, R 3, R 3 m, R 32$ and $R \overline{3} m$ with the vaule of CFOM 7.68, 25.36, 47.09, 47.09 and 31.27, respectively. The mean value of $\left|E^{2}-1\right|(1.213)$ also suggested that the space group is centrosymmetric, thus the space group $R \overline{3}$ with the smallest CFOM value is selected. A structural solutions with the $C 2 / m$ and $R \overline{3}$ space group were obtained for $\mathrm{VI}_{3}$ by intrinsic phasing methods using the program APEX $3 \frac{16}{\underline{1}}$ and the final refinement was completed with the SHELXL suite of programs $\underline{\underline{17}}$

Measurements of Physical Properties. The magnetic properties were measured using a Quantum Design magnetic property measurement system (MPMS-3). A piece of $\mathrm{VI}_{3}$ single crystal was mounted in the plastic straw with different orientations in order to measure the anisotropy of magnetization. Heat capacity measurements were performed using a Quantum Design physical property measurement system (PPMS-14T). Exfoliation of bulk $\mathrm{VI}_{3}$ was achieved using mechanical exfoliation with scotch tape and transferred onto a $90 \mathrm{~nm} \mathrm{SiO}{ }_{2}$ covered Si substrate. Atomic force microscopy was carried out using a Bruker Edge Dimension atomic force microscope.

Theoretical Calculation. The fully spin-polarized electronic structure calculations were carried out with the projector augmented wave (PAW) method 18 as imple-

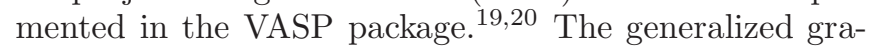
dient approximation (GGA) of Perdew-Burke-Ernzerhof (PBE) type ${ }^{21}$ was chosen for the exchange-correlation functional. The kinetic energy cutoff of the plane-wave basis was set to be $400 \mathrm{eV}$. The low-temperature $(40 \mathrm{~K})$ $R \overline{3}$ crystal structure with the experimental lattice constants (Table I) was adopted. Four typical intralayer magnetic configurations, $\stackrel{15}{=}$ i.e., the ferromagnetic (FM) state, the checkerboard antiferromagnetic (AFM) Néel state, the AFM stripy state, and the AFM zigzag state, were investigated. These magnetic configurations were studied with a $\sqrt{3} \times 1 \times 1$ orthorhombic supercell, which contains four $\mathrm{V}$ atoms in each layer, and a $4 \times 8 \times 4$ $k$-point mesh. The Fermi level was broadened by the Gaussian smearing method with a width of $0.05 \mathrm{eV}$. The electronic correlation effect among V $3 d$ electrons was in-
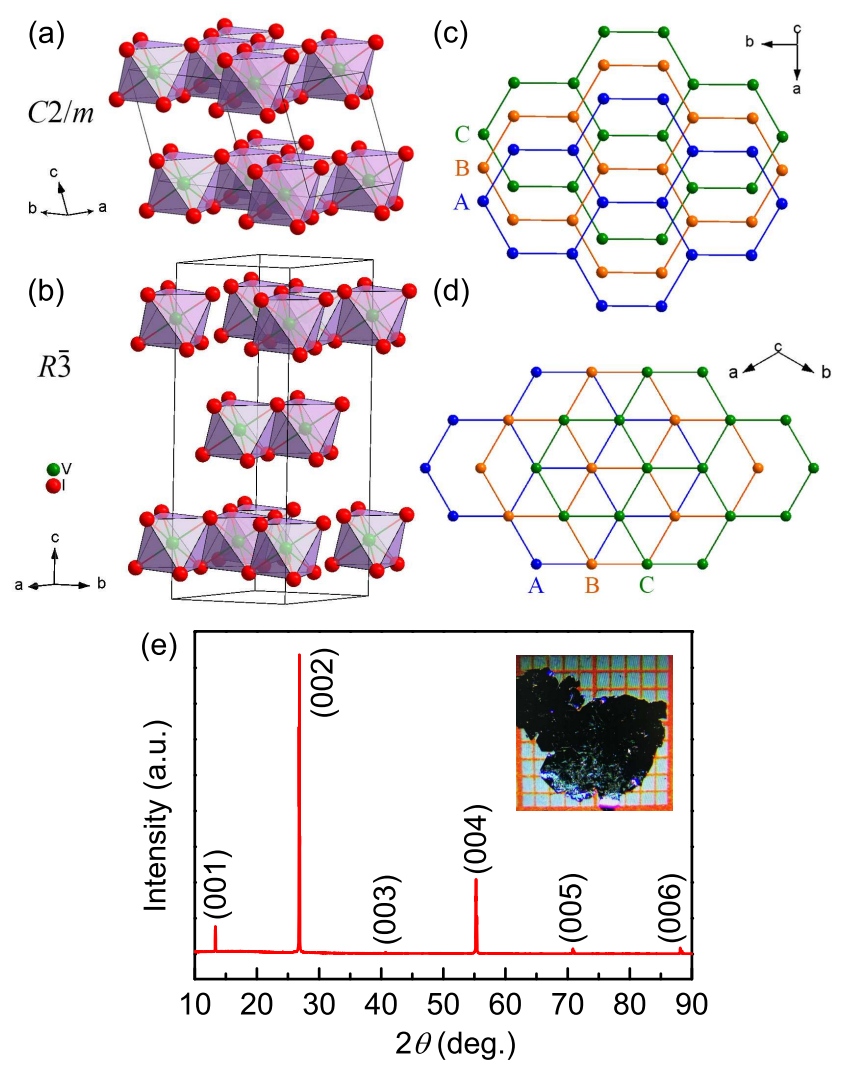

FIG. 1. (a) and (b) High- and low-temperature crystal structures of $\mathrm{VI}_{3}$, respectively. (c) and (d) Stacking pattern of the $\mathrm{V}$ layers in the high- and low-temperature structures of $\mathrm{VI}_{3}$, respectively. The labels of $\mathrm{A}, \mathrm{B}$ and $\mathrm{C}$ denote the different layers of $\mathrm{V}$ atoms. (e) XRD pattern of a $\mathrm{VI}_{3}$ single crystal. Inset: photo of typical $\mathrm{VI}_{3}$ single crystals on a $1 \mathrm{~mm}$ grid paper.

corporated by using the GGA + U formalism of Dudarev et al. $\underline{22}$ with the effective Hubbard $U$ of $3.68 \mathrm{eV} \stackrel{15}{\underline{1}}$ The $\mathrm{vdW}$ interactions between the $\mathrm{VI}_{3}$ layers were considered by adopting the optB86b-vdW functional. $\underline{23}$ The internal atomic positions were allowed to relax until all forces on atoms were smaller than $0.01 \mathrm{eV} / \AA$. The spin-orbit coupling (SOC) effect was included when the equilibrium atomic positions were obtained. The binding energy $E_{\mathrm{B}}$ was calculated by increasing the interlayer distance $d$ of a $R \overline{3}$ conventional cell ${ }^{24}$

\section{RESULTS AND DISCUSSION}

As shown in Table I and II, the crystal structure of $\mathrm{VI}_{3}$ at high temperature is not rhombohedral $\mathrm{BiI}_{3}$ type reported in previous study on the $\mathrm{VI}_{3}$ polycrystal, 25 but monoclinic $\mathrm{AlCl}_{3}$ type (space group $C 2 / m$, No. 12 ), similar to high-temperature structure of $\mathrm{CrX}_{3} ! \underline{14,26,27}$ For this structure (Fig. 1(a)), the $\mathrm{V}^{3+}$ cations are surrounded by six $\mathrm{I}^{-}$anions with an octahedral coordination. These edge-shared distorted octahedra form honeycomb layers in the $a b$ plane. The slabs of $\mathrm{VI}_{3}$ are stacked along the $c$ 
TABLE I. Crystallographic Data for $\mathrm{VI}_{3}$ at Different Temperatures.

\begin{tabular}{|c|c|c|c|}
\hline$T(\mathrm{~K})$ & 40 & 60 & 100 \\
\hline space group & $R \overline{3}$ & $R \overline{3}$ & $C 2 / m$ \\
\hline crystal system & trigonal & trigonal & monoclinic \\
\hline$a(\AA)$ & $6.8351(7)$ & $6.8325(6)$ & $6.8416(3)$ \\
\hline$b(\AA)$ & $6.8351(7)$ & $6.8325(6)$ & $11.8387(6)$ \\
\hline$c(\AA)$ & $19.696(2)$ & $19.6776(2)$ & $6.9502(4)$ \\
\hline$V\left(\AA^{3}\right)$ & $796.90(19)$ & $795.54(16)$ & $533.66(36)$ \\
\hline$Z$ & 6 & 6 & 4 \\
\hline dimens $\min / \operatorname{mid} / \max \left(\mathrm{mm}^{3}\right)$ & $0.03 / 0.28 / 0.37$ & $0.03 / 0.28 / 0.37$ & $0.03 / 0.28 / 0.37$ \\
\hline calcd density $\left(\mathrm{g} \mathrm{cm}^{-3}\right)$ & 5.397 & 5.406 & 5.372 \\
\hline abs coeff $\left(\mathrm{mm}^{-1}\right)$ & 19.117 & 19.149 & 19.031 \\
\hline$h$ & $-9 \leq h \leq 9$ & $-8 \leq h \leq 8$ & $-9 \leq h \leq 9$ \\
\hline$k$ & $-8 \leq k \leq 7$ & $-9 \leq k \leq 9$ & $-15 \leq k \leq 15$ \\
\hline$l$ & $-26 \leq l \leq 26$ & $-26 \mathrm{x} \leq l \leq 26$ & $-9 \leq l \leq 9$ \\
\hline reflns collected/unique/ $R$ (int) & $3799 / 442 / 0.0409$ & $4793 / 442 / 0.0471$ & $4901 / 681 / 0.0417$ \\
\hline data/params/restraints & $442 / 15 / 0$ & $442 / 15 / 0$ & $681 / 22 / 0$ \\
\hline GOF on $F^{2}$ & 1.228 & 1.287 & 1.025 \\
\hline$R$ indices (all data) $(R 1 / w R 2)^{a}$ & $0.0636 / 0.1691$ & $0.0502 / 0.1549$ & $0.0724 / 0.1828$ \\
\hline
\end{tabular}

TABLE II. Atomic Positions and Equivalent Isotropic Displacement Parameters $U_{\text {eq }}$ for $\mathrm{VI}_{3}$ at Different Temperatures.

\begin{tabular}{cccccc}
\hline atom & site & $x / a$ & $y / b$ & $z / c$ & $U_{\text {eq }}\left(\mathrm{A}^{2}\right)$ \\
$40 \mathrm{~K}$ & & & & & \\
$\mathrm{~V}$ & $6 c$ & $1 / 3$ & $2 / 3$ & $0.50035(11)$ & $0.0056(7)$ \\
$\mathrm{I}$ & $18 f$ & $0.00134(7)$ & $0.6509(2)$ & $0.42086(3)$ & $0.0074(5)$ \\
$60 \mathrm{~K}$ & & & & & \\
$\mathrm{~V}$ & $6 c$ & $1 / 3$ & $2 / 3$ & $0.50035(11)$ & $0.0052(7)$ \\
$\mathrm{I}$ & $18 f$ & $0.00143(8)$ & $0.6507(2)$ & $0.42086(3)$ & $0.0069(5)$ \\
$100 \mathrm{~K}$ & & & & & \\
$\mathrm{~V}$ & $4 g$ & 0 & $0.16658(18)$ & 0 & $0.0136(7)$ \\
$\mathrm{I} 1$ & $4 i$ & $0.2255(2)$ & 0 & $0.23895(15)$ & $0.0167(5)$ \\
$\mathrm{I} 2$ & $8 j$ & $0.25103(10)$ & $0.32498(8)$ & $0.23547(13)$ & $0.0163(5)$ \\
\hline
\end{tabular}

axis and there are vdW gaps between them. On the other hand, when decreasing temperature, there is a structural transition from monoclinic phase to rhombohedral one with space group $R \overline{3}$ (Tables I and II, Fig. 1(b)), same as $\mathrm{CrX}_{3}(\mathrm{X}=\mathrm{Cl}, \mathrm{Br}, \mathrm{I}) \stackrel{14,26,27}{ }$ The key difference between these two structures is the stacking patterns of $\mathrm{V}-\mathrm{I}$ slabs along the $c$ axis. For the monoclinic structure, the V-I slab shifts layer-by-layer along the $a$ axis. Because the $\beta$ is about $108.558^{\circ}$ and the $a$ and $c$ axial lattice parameters are 6.8416 and $6.9502 \AA$, the $a /\left[c \cos \left(180^{\circ}-\beta\right)\right]$ is 3.09 , i.e., the stacking is nearly the ABC fashion (Fig. 1(c)). For the rhombohedral structure (Fig. 1(b)), the stacking of $\mathrm{V}$-I slabs along the $c$ axis is exactly the ABC fashion, i.e., each V-I layer shifts the distance of $\mathrm{V}-\mathrm{V}$ bond $(\sim 3.95 \AA)$ along one edge of $\mathrm{V}$ honeycomb (the [110] direction) (Fig. 1(d)). This shift direction is distinctly different from that perpendicular to one edge of $\mathrm{V}$ honeycomb in the monoclinic structure. In contrast to the drastic change of stacking order, the intralayer structure is almost intact, except a slight decreased distortion of $\mathrm{V}$ honeycomb and V-I octahedron in rhombohedral structure. The bond lengths of V-I are spread between 2.7122 and $2.7195 \AA$ in monoclinic structure and narrow down to 2.7173 and $2.7179 \AA$ for the low-temperature phase (Table III). Moreover, in high-temperature structure, the $\mathrm{V}$ honeycomb is distorted with two bond lengths of $\mathrm{V}-\mathrm{V}$ (3.9442 and $3.9501 \AA$ ). It becomes undistorted in low-temperature structure and the single bond length of $\mathrm{V}-\mathrm{V}$ is about $3.9463 \AA$. In the XRD pattern of a $\mathrm{VI}_{3}$ single crystal (Fig. 1(e)), only the (00l) reflections of monoclinic structure can be observed, indicating that the surface of crystal is parallel to the $a b$ plane. The single crystals of $\mathrm{VI}_{3}$ show plate-like shapes (inset of Fig. 1(e)), consistent with the single crystal XRD pattern and the layered structure of $\mathrm{VI}_{3}$. The actual atomic ratio of $\mathrm{V}$ : I determined from the EDX analysis is $1.00: 2.90(1)$ when setting the content of $\mathrm{V}$ as 1 , in agreement with the nominal formula of $\mathrm{VI}_{3}$.

Figure 2(a) shows the temperature dependence of magnetic moment $M(T)$ of the $\mathrm{VI}_{3}$ single crystal at various fields in the $a b$ plane and along the $c$ axis. It can be clearly seen that $\mathrm{VI}_{3}$ exhibits a ferromagnetic transition at low temperature for both field directions. The Curie 
TABLE III. Selected Bond Lengths (in $\AA$ ) for $\mathrm{VI}_{3}$ at Different Temperatures.

\begin{tabular}{ccc}
\hline $40 \mathrm{~K}$ & & \\
$\mathrm{~V}-\mathrm{I}$ & $3 \times$ & $2.7143(14)$ \\
$\mathrm{V}-\mathrm{I}$ & $3 \times$ & $2.7179(14)$ \\
$60 \mathrm{~K}$ & & \\
V-I & $3 \times$ & $2.7118(15)$ \\
V-I & $3 \times$ & $2.7161(14)$ \\
$100 \mathrm{~K}$ & & \\
V-I2 & $2 \times$ & $2.7122(17)$ \\
V-I2 & $2 \times$ & $2.7138(8)$ \\
V-I1 & $2 \times$ & $2.7195(18)$ \\
\hline
\end{tabular}
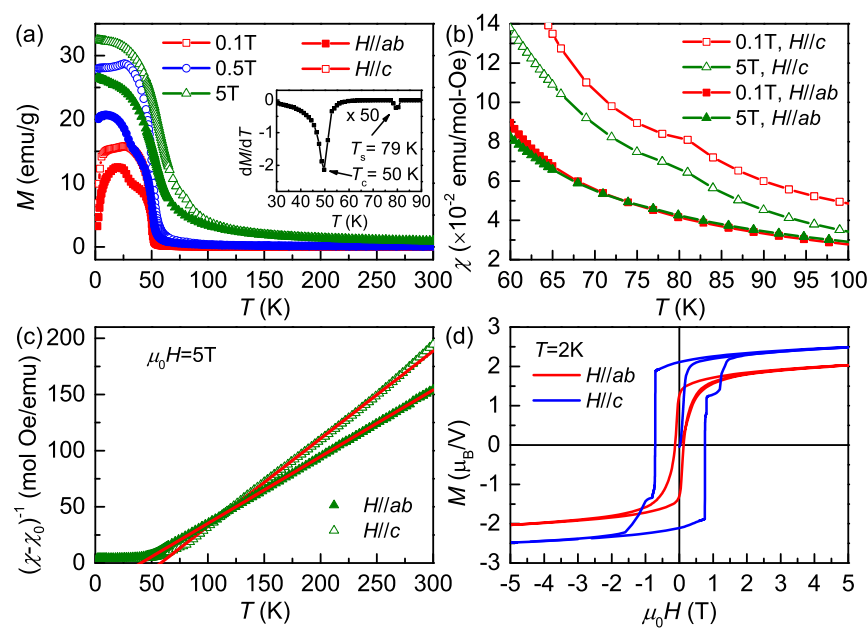

FIG. 2. (a) Temperature dependence of magnetic moment $M(T)$ of $\mathrm{VI}_{3}$ single crystal with zero-field-cooling (ZFC) mode at $\mu_{0} H=0.1,0.5$, and $5 \mathrm{~T}$ for $H \| a b$ and $H \| c$. Inset: $\mathrm{d} M(T) / \mathrm{d} T$ vs. $T$ at $\mu_{0} H=0.1 \mathrm{~T}$ for $H \| c$. (b) enlarged magnetic susceptibility $\chi(T)$ between $60-100 \mathrm{~K}$ at $\mu_{0} H=$ 0.1 and $5 \mathrm{~T}$ for both field directions. (c) Inverse magnetic susceptibility (points) and Curie-Weiss fits (solid lines) as a function of temperature at $\mu_{0} H=5 \mathrm{~T}$ for $H \| a b$ and $H \| c$. Fitted temperature-independent terms $\chi_{0}$ are subtracted. (d) Isothermal $M(H)$ curves at $2 \mathrm{~K}$ for both field directions.

temperature $T_{c}$ determined from the peak of $\mathrm{d} M / \mathrm{d} T$ at $\mu_{0} H=0.1 \mathrm{~T}$ for $H \| c$ is $50 \mathrm{~K}$ (inset of Fig. 2(a)), consistent with previous result $\underline{28}$ Another important feature is a kink appearing on the $\chi_{c}(T)$ curve at $T_{s} \sim 80 \mathrm{~K}\left(>T_{c}\right)$ and it can be seen more clearly from the small peak of $\mathrm{d} M / \mathrm{d} T$ at $79 \mathrm{~K}$ (inset of Fig. 2(a)). This kink does not shift with the variation of field (Fig. 2(b)). This temperature is in the temperature region of structural transition, thus the anomaly of $\chi_{c}(T)$ curve should originate from the change of crystal structure. In contrast, the $\chi_{a b}(T)$ curves do not show this behavior. It suggests that there is a more stronger coupling of the crystal structure to the magnetism along the stacking direction in $\mathrm{VI}_{3}$. Moreover, from the structure data shown in Table I, there is also remarkable change of the interlay spacing from 6.5888 $\AA\left[=c \sin \left(180^{\circ}-\beta\right)\right]$ at $100 \mathrm{~K}$ to $6.5592 \AA[=c / 3]$ at 60 $\mathrm{K}$. There is about $0.45 \%$ change when compared to the variation of $0.13 \%$ for the intralayer $a$-axial lattice parameters. Such huge changes of interlayer spacing could not be solely explained by the effect of conventional thermal contraction and the structural transition should take significant effect on it. It is worthy to mention that such subtle change accompanying by the sudden drop interlayer distance when crossing structural phase transition

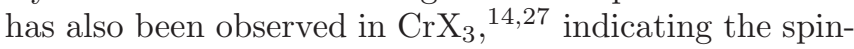
lattice coupling might be a common feature in layered magnetic halide compounds.

For both field directions, the $M(T)$ curves between 100 $\mathrm{K}$ and $300 \mathrm{~K}$ can be fitted very well using the modified Curie-Weiss law $\chi(T)=\chi_{0}+C /(T-\theta)$, where $\chi_{0}$ is a temperature-independent term including core diamagnetism, van Vleck paramagnetism as well as background signal of sample holder, $C$ is the Curie constant and $\theta$ is the Weiss temperature (Fig. 2(c)). The fitted $C$ and $\theta$ are 1.684(4) [1.281(8)] emu mol ${ }^{-1} \mathrm{Oe}^{-1} \mathrm{~K}$ and 40.5(1) [58.1(3)] $\mathrm{K}$ for $H \| a b[H \| c]$. The fitted positive values of $\theta$ for both field direction are close to the $T_{c}$, confirming the ferromagnetic interaction in $\mathrm{VI}_{3}$. The obtained values of $C$ correspond to an effective moment of $\mu_{\mathrm{eff}}=3.67(1)$ and $3.20(2) \mu_{\mathrm{B}} / \mathrm{V}$ for $H \| a b$ and $H \| c$, respectively. These values are somewhat larger than that of spin-only $\mathrm{V}^{3+}$ ions $\left(2.83 \mu_{\mathrm{B}} / \mathrm{V}\right)$. This suggests that the orbital moment of $\mathrm{V}^{3+}$ ions in $\mathrm{VI}_{3}$ might have some contribution on effective moment. On the other hand, when $T<T_{c}$, there is a kink locating at about $30 \mathrm{~K}$, and then a decrease of $M(T)$ at low temperature, which become invisible at high field. Such behavior is not clear at present and maybe originates from a possible magnetic glassy state at low temperature and low field region. Fig. 2(d) shows the magnetization loops for both field directions at $2 \mathrm{~K}$. The hysteresis loops for both field directions support the FM behavior when $T<T_{c}$. There is an obvious magnetic anisotropy at low field region. The initial magnetization curve becomes more quickly saturated for $H \| c$ than $H \| a b$, suggesting the easy axis is the $c$ axis. The coercive field for $H \| c$ is much larger than that for $H \| a b$ and it also indicates that the easy axis is along the $c$ axis. For $H \| a b$, the $M$ at $5 \mathrm{~T}$ is about $2.02 \mu_{\mathrm{B}} / \mathrm{V}$, which is close to the expected saturated moment of $g S=2 \mu_{\mathrm{B}} / \mathrm{V}$ for highspin state $\mathrm{V}^{3+}$ ion. But the $M(5 \mathrm{~T})$ for $H \| c$ is about 2.47 $\mu_{\mathrm{B}} / \mathrm{V}$, slightly larger than the expected value. It may be due to the anisotropy $g$ factor with unquenched orbital angular moment.

Figure 3 shows the temperature dependence of heat capacity $C_{p}(T)$ for $\mathrm{VI}_{3}$ single crystals measured between $T=2.2$ and $100 \mathrm{~K}$ at zero field. Clearly, there are two anomalies shown at $T \sim 50 \mathrm{~K}$ and $79 \mathrm{~K}$, in agreement with those temperatures in magnetization curve for $H \| c$. When increasing magnetic field, the $\lambda$-type peak at 49.5 $\mathrm{K}$ becomes broaden towards higher temperatures (Fig. $3(\mathrm{~b}))$. This is the typical behavior of FM transition un- 

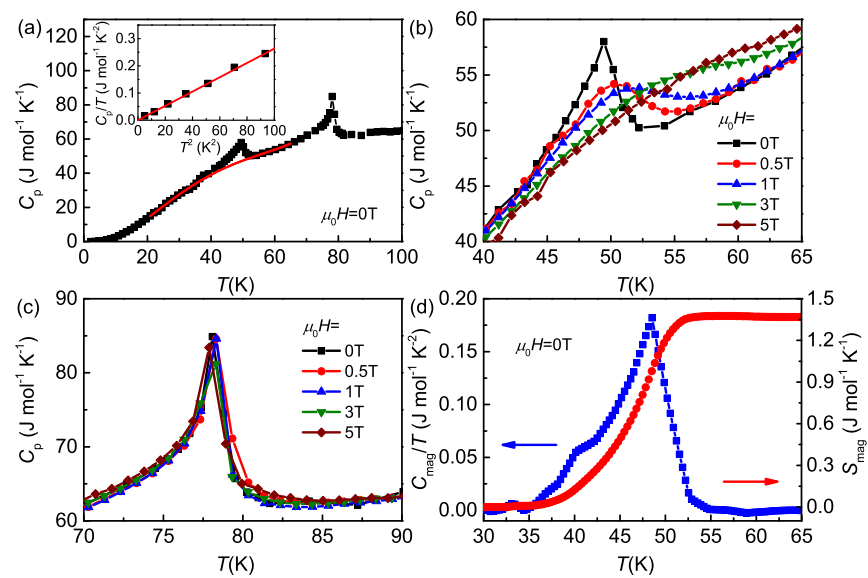

FIG. 3. (a) Temperature dependence of heat capacity $C_{p}(T)$ for $\mathrm{VI}_{3}$ single crystal at zero field. The red solid curve represents the lattice contribution, fitted by a polynomial. Inset: enlarged part at low temperature region. The red solid line represents the fit using the formula $C_{p}(T) / T=\beta T^{2}$. (b) The $C_{p}(T)$ as a function of $T$ near magnetic transition region at various fields. (c) Enlarged part of $C_{p}(T)$ near structural transition region at different fields. (d) Temperature dependence of magnetic specific heat $C_{\mathrm{mag}}(T)$ after subtracting the lattice contribution fitted by a polynomial. The right axis and its associated solid curve denote the magnetic entropy $S_{\text {mag }}(T)$ associated with the FM transition.

der field, and similar behaviors have been observed in $\mathrm{CrX}_{3} \stackrel{14,27}{=}$ Thus, it confirms the bulk nature of the longrange FM order below $T_{c}$ observed in the magnetization measurement. In contrast, the sharp peak at higher temperature $(\sim 79 \mathrm{~K})$ is insensitive to external field and there is almost no shift when the field is up to $5 \mathrm{~T}$ (Fig. 3(c)). It undoubtedly indicates that this anomaly of heat capacity is corresponding to the structural transition, consistent with the structural characterization. After subtraction of the phonon contribution $C_{\mathrm{ph}}(T)$ fitted using a polynomial from the total heat capacity (red line in the main panel of Fig. 3(a)), we obtain magnetic specific heat $C_{\mathrm{mag}}(T)$ and calculate the magnetic entropy $S_{\text {mag }}(T)$ using the formula $S_{\text {mag }}(T)=\int_{0}^{T} C_{\text {mag }}(T) / T d T$ (Fig. 3(d)). The derived $S_{\text {mag }}$ is about $1.37 \mathrm{~J} \mathrm{~mol}^{1} \mathrm{~K}^{-1}$ at $65 \mathrm{~K}$, which is much smaller than the expected value $(\sim 15 \% \operatorname{Rln}(2 S+1)=\mathrm{R} \ln 3)$ for $\mathrm{V}^{3+}$ ions with high spin state $(S=1)$. One of possibilities leading to this difference is the overestimation of $C_{\mathrm{ph}}(T)$, reducing the $S_{\mathrm{mag}}(T)$. Another possibility is that there is substantial fraction of magnetic entropy released above $T_{c}$ because of possible short-range or $2 \mathrm{D}$ magnetic correlations before long-range order forms. But it has to be mentioned that the Weiss temperature $\theta$ determined from the CurieWeiss law is close to $T_{c}$, implying the magnetic correlations/fluctuation should not so strong when $T>T_{c}$. Similar behaviors are also observed in $\mathrm{CrX}_{3} \cdot \underline{14,27}$, suggesting certain universal features in these $\mathrm{vdW}$ magnetic materials. Further theoretical and experimental studies are needed in order to understand these phenomena and

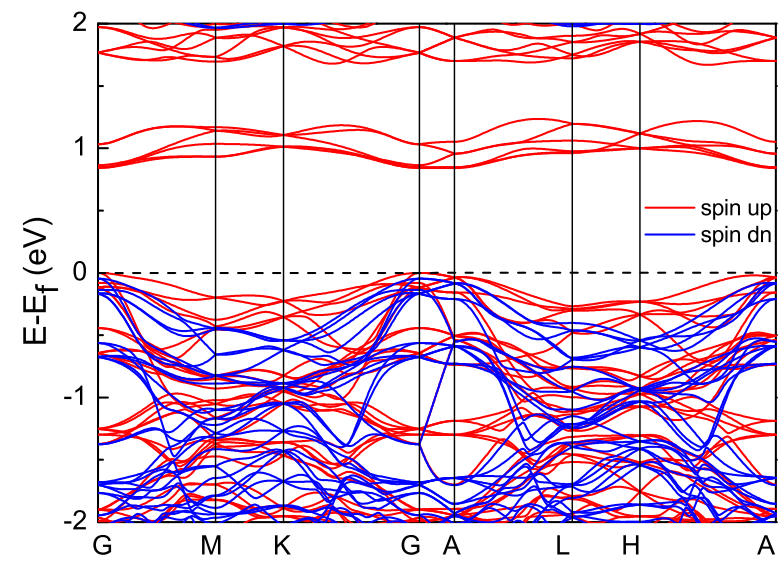

FIG. 4. Calculated electronic band structure of bulk $\mathrm{VI}_{3}$ in the ferromagnetic ground state along the high-symmetry paths in the Brillouin zone of the $R \overline{3}$ conventional cell.

the relationship between magnetism and low-dimensional structure. At the low temperature, the $C_{p}(T)$ curve can be fitted solely by a cubic term $\beta T^{3}$ (inset of Fig. 3(a)) because of the insulating feature of $\mathrm{VI}_{3}$ (the resistance is about $1 \mathrm{M} \Omega$ measured using ohmmeter at room temperature). the fitted value of $\beta=2.63(3) \mathrm{mJ} \mathrm{mol}^{-1} \mathrm{~K}^{-4}$, the Debye temperature is estimated to be $\Theta_{D}=144(1)$ $\mathrm{K}$ using the formula $\Theta_{D}=\left(12 \pi^{4} N R / 5 \beta\right)^{1 / 3}$. This is

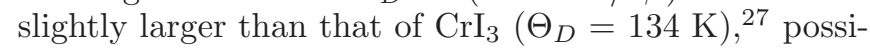
bly ascribed to the smaller atomic mass of $\mathrm{V}$ than $\mathrm{Cr}$.

To further investigate the magnetic and electronic properties of bulk $\mathrm{VI}_{3}$ in the $R \overline{3}$ structure at low temperature, we have carried out the spin-polarized firstprinciples calculations with the experimental lattice constants (Table I) and the relaxed internal atomic positions. By comparing the relative energies among four typical intralayer magnetic configurations (the FM order, the

(a)
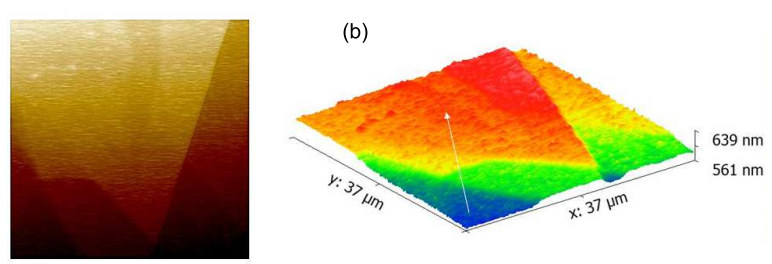

(c)
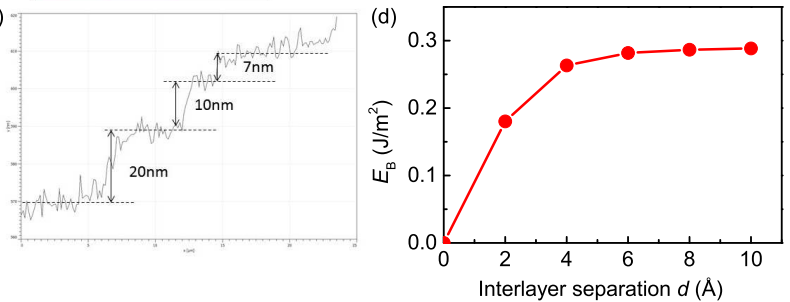

FIG. 5. (a) AFM image of a cleaved thin flake of $\mathrm{VI}_{3}$ crystal. (b) Height profile of whole flake. The different colors represent the various heights of surface. (c) Height profile across several step edges along the direction shown by the arrow in (b). (d) Calculated binding energy $E_{\mathrm{B}}$ as a function of the interlayer separation $d$. 
checkerboard AFM Néel order, the AFM stripy order,

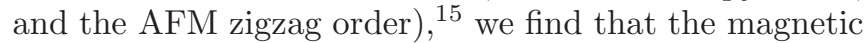
ground state of bulk $\mathrm{VI}_{3}$ is the $\mathrm{FM}$ order, being energetically at least $3.8 \mathrm{meV} / \mathrm{V}$ lower than those of the AFM orders. Moreover, our calculations demonstrate that bulk $\mathrm{VI}_{3}$ prefers a weak FM interlayer coupling. As to the electronic structure, without including the Hubbard $U$, our calculation predicts that $\mathrm{VI}_{3}$ should be metallic, different from the insulating behavior observed in experiment. When considering the effect of Hubbard $U$ (3.68

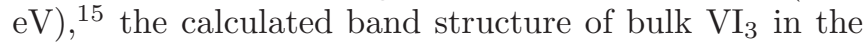
FM ground state shows a direct band gap of $\sim 0.84 \mathrm{eV}$ (Fig. 4). This calculation result suggests that bulk $\mathrm{VI}_{3}$ is a FM Mott insulator.

Figure 5(a) displays an atomic force microscopy image of a cleaved thin flake of a $\mathrm{VI}_{3}$ crystal and there are several step edges observed on the flake. As shown in Fig. 5(b), the total thickness of this flake is about tens of nanometers and the area along the $a b$ plane is rather large $\left(\sim 40 \times 40 \mu \mathrm{m}^{2}\right)$. The hight profile across an edge measured along the white arrow in Fig. 5(b) consists of three steps with heights of 20, 10 and $7 \mathrm{~nm}$ (Fig. 5(c)). The minimal step height $(\sim 7 \mathrm{~nm})$ is about 10 unit cells of monoclinic structure of $\mathrm{VI}_{3}\left(h=c \sin \left(180^{\circ}-\beta\right)=\right.$ $6.5888 \AA$ ). It suggests that $\mathrm{VI}_{3}$ is easy to be exfoliated down to few layers. The binding energy $E_{\mathrm{B}}$ was calculated by increasing the interlayer distance $d$ of $\mathrm{V}_{3}$ in a $R \overline{3}$ conventional cell $\stackrel{24}{\underline{*}}$ The calculated $E_{\mathrm{B}}$ as a function of interlayer separation $d$ is shown in Fig. 5(d). The $E_{\mathrm{B}}$ increases quickly with increasing $d$ and then converges to the saturation value $0.29 \mathrm{~J} / \mathrm{m}^{2}\left(18 \mathrm{meV} / \AA^{2}\right)$ when $d$ is lager than $6 \AA$. This value is in good agreement with previous studies on most vdW crystals $\left(E_{\mathrm{B}}=13-21\right.$ $\left.\mathrm{meV} / \AA^{2}\right) \stackrel{24}{\underline{\underline{2}}}$ Thus it confirms the weak interlayer inter- action in $\mathrm{VI}_{3}$, i.e., its easy exfoliation.

\section{CONCLUSION}

In summary, we synthesized $\mathrm{VI}_{3}$ single crystals successfully and studied the structural and physical properties of novel vdW magnetic compound $\mathrm{VI}_{3}$ in detail. The structure of $\mathrm{VI}_{3}$ at high temperature is monoclinic and changes to rhombohedral at $T_{s}=79 \mathrm{~K}$. The long-range FM order appears at $T_{c}=50 \mathrm{~K}$ with the saturate moment close to $2 \mu_{\mathrm{B}} / \mathrm{V}$. It suggests that the $\mathrm{V}^{3+}$ ion in $\mathrm{VI}_{3}$ is at high-spin state with $S=1$. Theoretical calculation implies that $\mathrm{VI}_{3}$ might be a FM Mott insulator. Moreover, it is easy to be cleaved down to few layers, thus it is a promising candidate of $2 \mathrm{D}$ ferromagnet with $S=$ 1. Exploration of magnetic vdW materials with different magnetic moments will not only expand the family of $2 \mathrm{D}$ magnets but also deepen the understanding of the relationship between dimensionality and degrees of freedom for spin space.

\section{ACKNOWLEDGEMENTS}

This work was supported by the National Key R\&D Program of China (Grants No. 2015CB921400, 2016YFA0300504 and 2017YFA0302903), the National Natural Science Foundation of China (Grants No. 11574394, 11774423, 11774424 and 11822412), the Fundamental Research Funds for the Central Universities, and the Research Funds of Renmin University of China (Grants No. 15XNLF06, 15XNLQ07 and 18XNLG14). Computational resources were provided by the Physical Laboratory of High Performance Computing at Renmin University of China.

$\dagger$ These authors contributed equally to this work.

* zhangxiaobupt@bupt.edu.cn, kliu@ruc.edu.cn, hlei@ruc.edu.cn
${ }^{1}$ K. S. Novoselov, A. K. Geim, S. V. Morozov, D. Jiang, Y. Zhang, S. V. Dubonos, I. V. Grigorieva, and A. A. Firsov, Science 306, 666-669 (2004).

${ }^{2}$ K. F. Mak, C. Lee, J. Hone, J. Shan, and T. F. Heinz, Phys. Rev. Lett. 105, 136805 (2010).

3 J. M. Lu, O. Zheliuk, I. Leermakers, N. F. Q. Yuan, U. Zeitler, K. T. Law, and J. T. Ye, Science 350, 1353-1357 (2015).

4 X. Xi, Z. Wang, W. Zhao, J.-H. Park, K. T. Law, H. Berger, L. Forró, J. Shan, and K. F. Mak, Nat. Phys. 12, 139-143 (2015).

${ }^{5}$ K. Chang, J. Liu, H. Lin, N. Wang, K. Zhao, A. Zhang, F. Jin, Y. Zhong, X. Hu, W. Duan, Q. Zhang, L. Fu, Q.-K. Xue, X. Chen, and S.-H. Ji, Science 353, 274-278 (2016).

6 N. D. Mermin and H. Wagner, Phys. Rev. Lett. 17, 11331136 (1966).

7 C. Gong, L. Li, Z. Li, H. Ji, A. Stern, Y. Xia, T. Cao, W. Bao, C. Wang, Y. Wang, Z. Q. Qiu, R. J. Cava, S. G.
Louie, J. Xia, and X. Zhang, Nature 546 265-269 (2017).

8 B. Huang, G. Clark, E. Navarro-Moratalla, D. R. Klein, R. Cheng, K. L. Seyler, D. Zhong, E. Schmidgall, M. A. McGuire, D. H. Cobden, W. Yao, D. Xiao, P. JarilloHerrero, and X. Xu, Nature 546, 270-273 (2017).

9 S. Jiang, L. Li, Z. Wang, K. F. Mak, and J. Shan, Nat. Nano. 13, 549-553 (2018).

10 Y. Deng, Y. Yu, Y. Song, J. Zhang, N. Z. Wang, Y. Z. Wu, J. Zhu4, J. Wang, X. H. Chen, and Y. Zhang, Nature 563, 94-99 (2018).

11 D. Zhong, K. L. Seyler, X. Linpeng, R. Cheng, N. Sivadas, B. Huang, E. Schmidgall, T. Taniguchi, K. Watanabe, M. A. McGuire, W. Yao, D. Xiao, K.-M. C. Fu, and X. Xu, Sci. Adv. 3, e1603113 (2017).

12 M.-W. Lin, H. L. Zhuang, J. Yan, T. Z. Ward, A. A. Puretzky, C. M. Rouleau, Z. Gai, L. Liang, V. Meunier, B. G. Sumpter, P. Ganesh, P. R. C. Kent, D. B. Geohegan, D. G. Mandrus, and K. Xiao, J. Mater. Chem. C 4, 315-322 
(2016)

13 W.-B. Zhang, Q. Qu, P. Zhu, and C.-H. Lam, J. Mater. Chem. C 3, 12457-12468 (2015).

14 M. A. McGuire, G. Clark, S. KC, W. M. Chance, G. E. Jellison, Jr., V. R. Cooper, X. Xu, and B. C. Sales, Phys. Rev. Mater. 1, 014001 (2017).

15 J. He, S. Ma, P. Lyu, and P. Nachtigall, J. Mater. Chem. C 4, 2518-2526 (2016).

16 APEX3 Ver. 2015, Bruker AXS, Inc., Madison, WI (2015).

17 G. M. Sheldrick, Acta Crystallogr. A 64, 112-122 (2008).

18 P. E. Blöchl, Phys. Rev. B 50, 17953-17979 (1994); G. Kresse and D. Joubert, Phys. Rev. B 59, 1758-1775 (1999).

19 G. Kresse and J. Hafner, Phys. Rev. B 47, 558-561(R) (1993); J. Phys.: Condens. Matter 6, 8245-8257 (1994).

20 G. Kresse and J. Furthmüller, Comput. Mater. Sci. 6, 1550 (1996); Phys. Rev. B 54, 11169-11186 (1996).
21 J. P. Perdew, K. Burke, and M. Ernzerhof, Phys. Rev. Lett. 77, 3865-3868 (1996).

22 S. L. Dudarev, G. A. Botton, S. Y. Savrasov, C. J. Humphreys, and A. P. Sutton, Phys. Rev. B 57, 1505-1509 (1998).

23 J. Klimeš, D. R. Bowler, and A. Michaelides, J. Phys.: Cond. Matt. 22, 022201 (2010).

${ }^{24}$ T. Björkman, A. Gulans, A. V. Krasheninnikov, and R. M. Nieminen, Phys. Rev. Lett. 108, 235502 (2012).

${ }^{25}$ K. O. Berry, R. R. Smardzewski, and R. E. McCarley, Inorg. Chem. 130, 1994-1997 (1969).

26 B. Morosin and A. Narath, J. Chem. Phys. 40, 1958-1967 (1964).

27 M. A. McGuire, H. Dixit, V. R. Cooper, and B. C. Sales, Chem. Mater. 27, 612-620 (2015).

28 J. Wilson, C. Maule, P. Strange, and J. Tothill, J. Phys. C: Solid State Phys. 20, 4159-4167 (1987). 